\title{
The Path to Better Understanding Stochastics in EUV Photoresist
}

\author{
Danilo De Simone $^{1 *}$, Yannick Vesters ${ }^{1,2}$, and Geert Vandenberghe ${ }^{1}$ \\ ${ }^{1}$ IMEC, Kapeldreef 75, 3001 Leuven, Belgium \\ ${ }^{2}$ KU Leuven, dept. Chemistry, Celestijnenlaan, 200F, Leuven, Belgium \\ *danilo.desimone@imec.be
}

\begin{abstract}
Although in the last year the development of extreme ultraviolet (EUV) lithography has allowed to push the lithographic performance of the EUV photoresists, the high-volumemanufacturing (HVM) requirement to have a cost-effective low exposure dose photoresist $\left(<20 \mathrm{~mJ} / \mathrm{cm}^{2}\right)$ remains a big challenge and patterning stochastic defectivity at nano-scale currently still is the major limiting factor of the lithographic process window of EUV resist when looking at tight pitches below $40 \mathrm{~nm}$. To get a better understanding of stochastic patterning failures for such tight pitches it is crucial to quantify them already at the early stage of R\&D resist screening phase. In this work we describe a new way of looking at the performance of EUV photoresists. We present the EUV resist nano-failure characterization results when printing dense line-space pattern logic use case on the ASML NXE3300 full field EUV scanner. We analyze through dense pitches three chemically amplified resists with different sensitivity, we quantify the nano-bridges and we discuss potential root causes from photon-shot-noise, imaging and material standpoint.
\end{abstract}

Keywords: EUV, EUVL, Stochastics, Nano-failures, Nano-bridges, Photoresist

\section{Introduction}

In the last year, the continuous efforts on the development of extreme ultraviolet (EUV) lithography has allowed to push the lithographic performance of the EUV photoresists on the ASML NXE:3300 full field exposure tool. Today imec N7 node (equivalent to foundry N5) is the first scaling node at which industry will likely insert EUV into production which will bring a reduction in processing steps therefore reducing total cost of ownership [1], increasing yield and reducing time to ramp. However, the high-volume-manufacturing (HVM) requirement to have a cost-effective low exposure dose photoresist $\left(20-30 \mathrm{~mJ} / \mathrm{cm}^{2}\right.$ exposure dose range) remains a big challenge and pattern defectivity at nano-scale is the major limiting factor of the lithographic process window of EUV resist when looking at tight pitches below 40nm. Recent studies on pitch $32 \mathrm{~nm}$ dense line space patterning and pitch $36 \mathrm{~nm}$ dense orthogonal contact hole patterning have shown pattern defectivity as nanobridges and broken-lines, and merging or closing, respectively [2,3]. Therefore, it is extremely important to be effective at the early characterization stage of the EUV resist to quantify the nano-failures induced by a stochastic EUV patterning regime, the random nature of the lightmatter interaction and consequent chemical reactions. To do that we have used for the EUV resist characterization a new metric to count stochastic printing failures [4], as to provide an early stage assessment on the patterning fidelity of the examined resist. The new metric to count pattern failures have been applied on three EUV chemically amplified resists with different sensitivity with specific focus on nano-bridge defects type for narrow dense line-space pitches as $40 \mathrm{~nm}$ and below. The experimental setup and the results of such a study are described and discussed in the next paragraphs.

\section{Experimental}

The EUV resist characterization was carried out by printing 1:1 line-space from $40 \mathrm{~nm}$ to $14 \mathrm{~nm}$ halfpitch using the same mask and exposure illumination. A $300 \mathrm{~mm}$ TEL clean track Lithius 
Pro $Z^{\mathrm{TM}}$ interfaced with the ASML NXE3300B full field EUV scanner was used. Two different illumination modes were used: a customized one, like dipole 90X and a standard quasar MP45. High resolution chemically amplified resists (CARs) were selected for this study with different sensitivity: Low Dose (LD), Mid Dose (MD), High Dose (HD). The optimum exposure dose and focus offset was picked up for each investigated pitch through a focus-exposure matrix to get equal 1:1 line-space pattern on wafer. The details of each resist are reported in Table 1. Hitachi CG5000 scanning electron microscope (CD-SEM) was used to collect top-down images and to measure the CD.

Table 1. Description of the chemically amplified resists used in this study.

\begin{tabular}{c|cccc}
\hline $\begin{array}{c}\text { Resist } \\
\text { name }\end{array}$ & Dose regime & FT & vendor & substrate \\
\hline LD & $\sim 20 \mathrm{~mJ} / \mathrm{cm}^{2}$ & $30 \mathrm{~nm}$ & A & Organic underlayer \\
MD & $\sim 30 \mathrm{~mJ} / \mathrm{cm}^{2}$ & $30 \mathrm{~nm}$ & A & Organic underlayer \\
HD & $-45 \mathrm{~mJ} / \mathrm{cm}^{2}$ & $35 \mathrm{~nm}$ & A & Optimum spin-on-glass \\
MD-2 & $\sim 30 \mathrm{~mJ} / \mathrm{cm}^{2}$ & $35 \mathrm{~nm}$ & B & Organic underlayer \\
HD-2 & $-45 \mathrm{~mJ} / \mathrm{cm}^{2}$ & $35 \mathrm{~nm}$ & C & Organic underlayer \\
\hline
\end{tabular}

The CD-SEM setting used to collect the images for nano-bridge defect count is reported in table 2 . Stochastic nano-failures were quantified using IMEC in house software Stochalis. This software package does an automatic assessment of top down CDSEM images based on the brightness of the pixels. The failures are quantified with the "pixel not OK" (pixNOK) metric, which essentially represents the failure probability: pixNOK= ¿pixels in failure / $\sum$ pixels inspected. A bridge becomes apparent when at least 4 consecutive pixels are failing (Fig. 1), corresponding to $3.2 \mathrm{~nm}$.

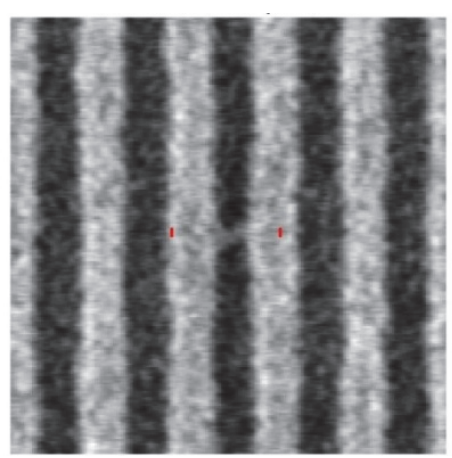

Fig. 1. Example on $16 \mathrm{~nm}$ half-pitch of a nano-bridge when 4 pixels ( $3.2 \mathrm{~nm}$ vertical height) fail.
Table 2. Settings to collect CDSEM top-down image for nano-bridge failure count.

\begin{tabular}{cccccccc}
\hline $\begin{array}{c}\text { \# of } \\
\text { images } \\
\text { per resist } \\
\text { and half- } \\
\text { pitch }\end{array}$ & Voltage & pA & Frame & Field size & $\begin{array}{c}\text { \# of pixel } \\
\text { per image }\end{array}$ & Pixel size & $\begin{array}{c}\text { Total } \\
\text { inspected } \\
\text { area per } \\
\text { resist and } \\
\text { half pitch }\end{array}$ \\
\hline 100 & 500 & 8 & 16 & $\begin{array}{c}1.638 \mu \mathrm{mx} \\
1.638 \mu \mathrm{m}\end{array}$ & $\begin{array}{c}2048 \mathrm{x} \\
2048\end{array}$ & $0.8 \mathrm{~nm}$ & $268 \mu \mathrm{m}^{2}$ \\
\hline
\end{tabular}

Table 3. Total number of pixels tested for all the trenches, detection limit and total number of trenches taken for each dense line-space pitch when analyzing 100 images collected on CD-SEM with the setting reported in Table 2.

\begin{tabular}{c|ccc}
\hline $\begin{array}{c}\text { half pitch } \\
\text { LS 1:1 }\end{array}$ & $\begin{array}{c}\text { Total Pixels tested for all } \\
\text { trenches (100 images) }\end{array}$ & $\begin{array}{c}\text { Detection } \\
\text { Limit }\end{array}$ & $\begin{array}{c}\text { \# of trenches } \\
\text { measured }\end{array}$ \\
\hline $\mathbf{1 4 H P}$ & $1.118 \mathrm{E}+07$ & $8.941 \mathrm{E}-08$ & 5592 \\
$\mathbf{1 5 H P}$ & $1.042 \mathrm{E}+07$ & $9.601 \mathrm{E}-08$ & 5208 \\
$\mathbf{1 6 H P}$ & $9.662 \mathrm{E}+06$ & $1.035 \mathrm{E}-07$ & 4831 \\
$\mathbf{1 7 H P}$ & $9.114 \mathrm{E}+06$ & $1.097 \mathrm{E}-07$ & 4557 \\
$\mathbf{1 8 H P}$ & $8.604 \mathrm{E}+06$ & $1.162 \mathrm{E}-07$ & 4302 \\
$\mathbf{2 0 H P}$ & $7.770 \mathrm{E}+06$ & $1.287 \mathrm{E}-07$ & 3885 \\
$\mathbf{2 2 H P}$ & $7.153 \mathrm{E}+06$ & $1.290 \mathrm{E}-07$ & 3500 \\
$\mathbf{2 5 H P}$ & $6.176 \mathrm{E}+06$ & $1.287 \mathrm{E}-07$ & 3088 \\
$\mathbf{2 7 H P}$ & $5.800 \mathrm{E}+06$ & $1.724 \mathrm{E}-07$ & 2900 \\
$\mathbf{4 0 H P}$ & $5.046 \mathrm{E}+06$ & $1.487 \mathrm{E}-07$ & 2000 \\
\hline \multicolumn{2}{|}{}
\end{tabular}

As the number of trenches present in one SEM image is pitch-dependent, the total number of inspected trenches and the total number of pixels tested are reported for each dense line-space pitch in Table 3, together with the detection limit $\left(1 / \sum\right.$ pixels inspected) when analyzing 100 top-down images. Reducing this detection limit of one order of magnitude would require 1000 images, bringing a practical limit regarding CD-SEM tool time usage.

Rigorous lithographic simulator ProLITH (from KLA-Tencor) version 6.0 was used to calculate the number of photons absorbed in the resist. A calibrated ProLITH resist model developed at imec was used in stochastic mode. For each pitch where Stochalis measurement was realized an equivalent simulation was run with a simulation region having a width of one pitch and a length of $1.638 \mu \mathrm{m}$ (equivalent to the trench length captured by one SEM image). Within this simulated region, the number of photon absorbed within the trench was calculated considering the trench width as half the pitch. To evaluate the variability of photon absorption along the trench, we defined a bridge volume as half-pitch $\times$ resist thickness $\times 3.6 \mathrm{~nm}$. Along each simulated trench, we measured the number of photons absorbed within that bridge volume and obtained average values and variability (10,000 nano-bridge volumes simulated per pitch). 


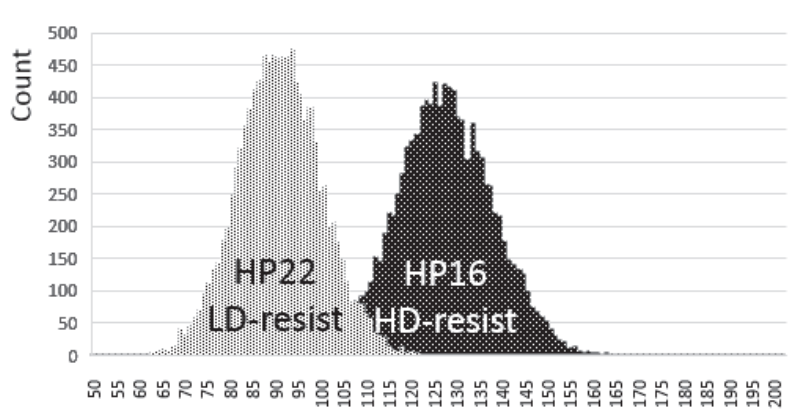

Number of photons absorbed per bridge volume

Fig. 2. Histogram of two distributions for 10,000 simulations of the number of photons absorbed in a bridge volume for LD and HD resists at $27.4 \mathrm{~mJ} / \mathrm{cm}^{2}$ and $45.4 \mathrm{~mJ} / \mathrm{cm}^{2}$ dose-to-size, respectively. The dose and the pitch influence the shape of the gaussian distribution. The bin width is 1 photon.

As example, the histogram of two distributions are presented in Fig. 2, showing an average of 91.4 photons absorbed per bridge volume for LD-resist at half-pitch $22 \mathrm{~nm}$ (std. dev. of 8.99), against an average of 127.1 photons per bridge volume for HD-resist at half-pitch $16 \mathrm{~nm}$ (std. dev. of 10.39).

\section{Results and discussion}

The nano-bridge defect count as pixNOK metric for the three resists LD, MD and HD is reported in Fig. 3 as function of the 1:1 line-space half-pitch.

The failure count is clearly modulated by the exposure dose and as soon as the resolution is pushed to reach $14 \mathrm{~nm}$ half-pitch the failure count increases for all three resists. The LD resist is the worse resist in terms of nano-bridge count. The HD resist with lower sensitivity (higher exposure dose) shows a lower pixNOK ratio compared to the other two resists; however, HP16, HP17, HP18, would require an increase in statistics to better distinguish them in failure count, as only one bridge is captured within the inspected area for these cases. It is worth noting that for HP15 and HP14 other failure modes like line collapse start to be dominant compared to the nano-failures but it is hard to decouple and separately quantify the two phenomena.

The impact of the illumination source on the failure count was tested on the MD resist looking at the nano-bridge type defect on 1:1 line space $32 \mathrm{~nm}$ pitch and comparing two illumination modes. The result is reported in Fig. 4 and it shows that by relaxing the illuminator from the dipole-like $90 \mathrm{X}$ to the quasar MP45, the nano-bridge count increases.

Besides the exposure dose, the pattern resolution and the illumination source, the resist chemistry platform matters as well when looking at nanofailures. In Fig. 5 a second resist (HD-2) that works in a similar dose regime as HD resist $\left(\sim 45 \mathrm{~mJ} / \mathrm{cm}^{2}\right)$ was examined and compared to HD resist looking at the failure count as function of the critical dimension (CD) of the trench. HD-2 resist gives a higher nano-bridge count with respect to HD for all three trench CDs taken into account.

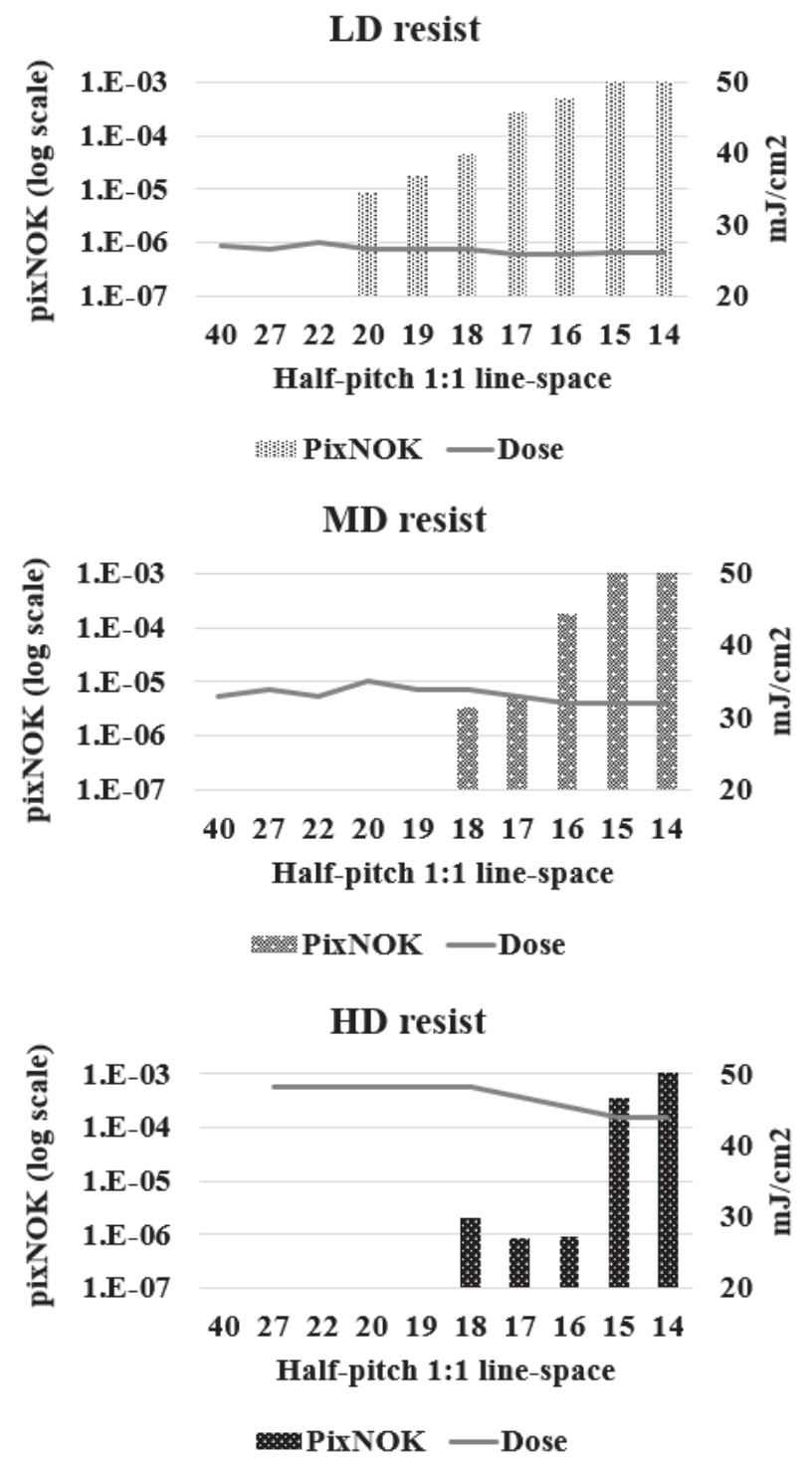

Fig. 3. Nano-bridge quantification for LD, MD and HD resists through 1:1 LS pitch for a same inspection area of $268 \mu^{2}$. The highest count is for LD resist, having a dose regime of $\sim 25 \mathrm{~mJ} / \mathrm{cm}^{2}$. The lowest count is for HD resist that has a dose of $\sim 45 \mathrm{~mJ} / \mathrm{cm}^{2}$. Half-pitch with no bar means that no failure was observed at the detection limit (Table 3). 


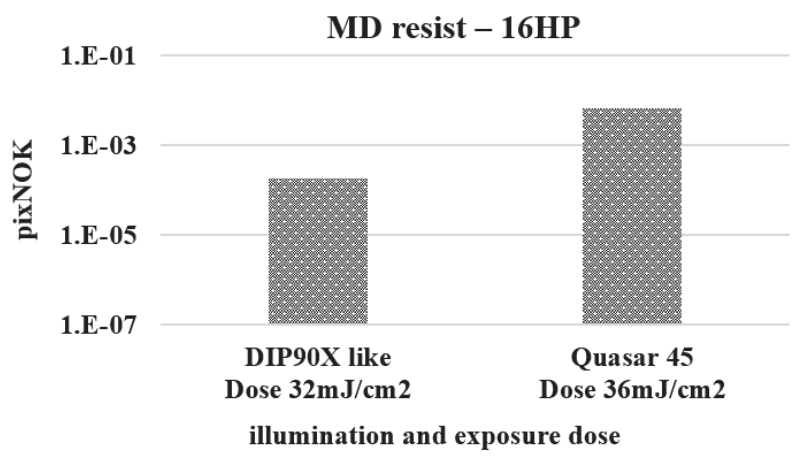

Fig. 4. Nano-bridge count as pixNOK metric for MD resist on 16HP for two illumination modes. The dipole illumination gives lower defect count confirming that the aerial image matters when looking at pattern nanofailures.

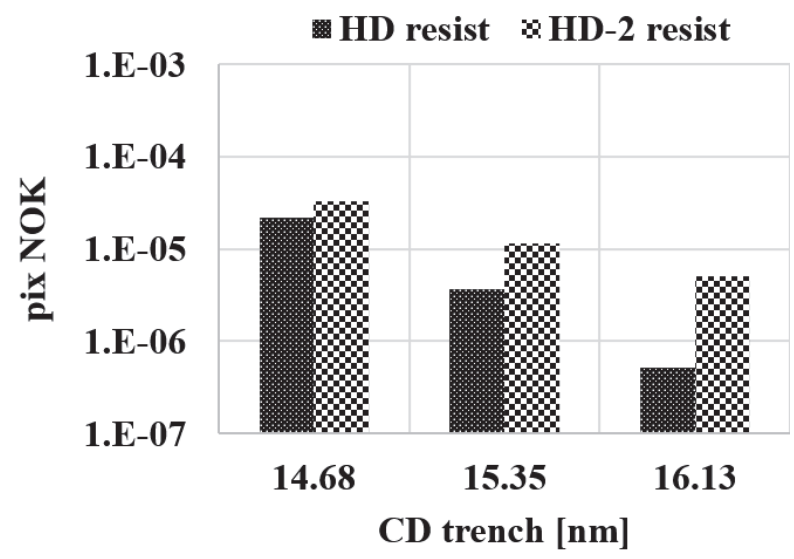

Fig. 5. Nano-bridge count as PixNOK metric for HD and HD-2 resist on $16 \mathrm{~nm}$ hp and three $\mathrm{CD}$ trench size. The HD resist gives lower defect count confirming that nanobridges are also driven by the resist formulation as expected.

The nano-bridge failure count was investigated from a different prospective by correlating the pixNOK ratio versus the absorbed photons in a trench $1.638 \mu \mathrm{m}$ long for the three resists (LD, MD and HD) at multiple pitches. The results are shown in Fig. 6. The pixNOK ratio correlates with the number of absorbed photons in the trench, but it is important to highlight the fact that to push down the pixNOK count for a given pitch it is needed to change the resist chemistry (from LD to HD resist). This material change requires a higher exposure dose but it allows to print with a lower nano-bridge count while keeping the same 1:1 line space pitch.

Moreover, by increasing the exposure dose the variability of the absorbed photons inside the bridge volume (see the experimental paragraph for the definition) is significantly reduced. In the case of 16 $\mathrm{nm} \mathrm{hp} \mathrm{(Fig.} \mathrm{7)} \mathrm{such} \mathrm{a} \mathrm{variability} \mathrm{is} \mathrm{reduced} \mathrm{by} 24 \%$ (from $75 \%$ to $57 \%$ ), thus reducing photon-shotnoise. Furthermore, that a potential bridge due to local lack of photons can be more easily compensated by the resist chemistry for a high exposure dose resist than for a low exposure dose resist. The three dots inside the circle in Fig. 6 intends to indicate that these three pitches seem comparable in terms of nano-bridge count, but they are close to the detection limit, and a larger statistic would be needed to better assess these dense features.

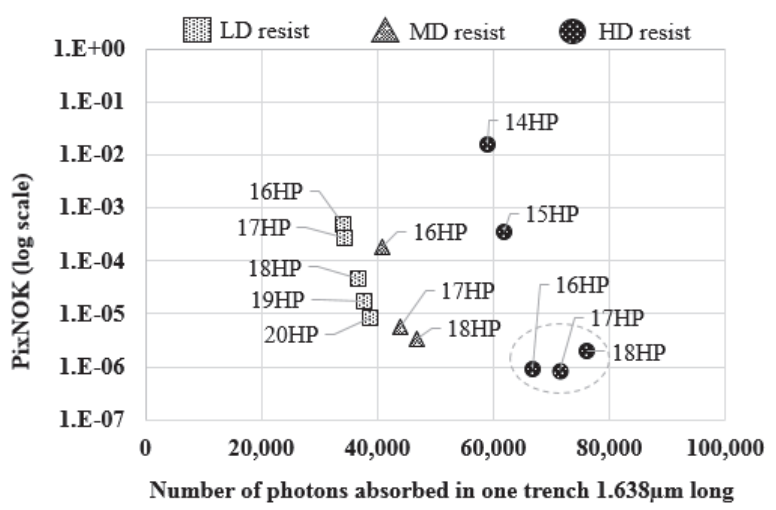

Fig. 6. Nano-bridge count as PixNOK metric for LD, MD and $\mathrm{HD}$ resists as function of the absorbed photons in the trench for multiple dense line space pitches.

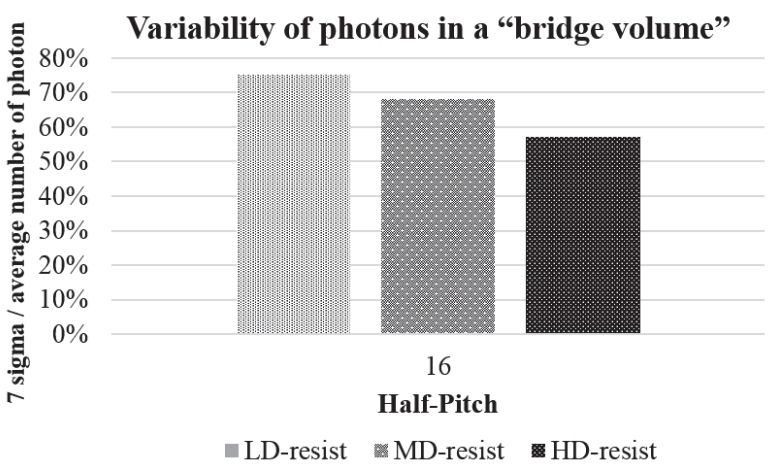

Fig. 7. Variability of photons as 7 sigma over the average of the absorbed photons in a trench long $1.638 \mu \mathrm{m}$ for the three resist LD, MD, HD on $16 \mathrm{~nm}$ half-pitch. The HD resist that requires higher dose has lower photon variability in the considered bridge volume.

\section{Conclusions}

In this work we have applied a new way of looking at the performance of EUV photoresists when pushing their resolution on the ASML NXE3300 full field EUV scanner. We have analyzed several advanced EUV chemically amplified resists by looking at nano-bridge type defects. We have looked at dense line space pattern from $40 \mathrm{~nm}$ to 14 $\mathrm{nm}$ half-pitch and we have quantified the nano- 
bridges considering resist sensitivity, resist platform, imaging and photo-shot-noise. The result shows that the low exposure dose and the tight resolution drive the nano-bridge failures, further modulated by the imaging quality together with the resist design.

Higher pattern resolution requires lower sensitivity resist to push down the nano-bridges. Lower sensitivity resist requires a different CAR chemistry. A different resist chemistry requires higher exposure dose. The higher the exposure dose, the lower the photon variability and the lack of photons in a "bridge volume" that can cause a nanobridge defect.

Today the best CAR that can print $16 \mathrm{~nm}$ halfpitch is working in an exposure dose regime of 45 $\mathrm{mJ} / \mathrm{cm}^{2}$ and it delivers $\sim 5 \times 10^{-7}$ pixNOK ratio at the best exposure conditions. Although it could be considered as a good result it must be improved since it is equivalent to approximately 1 nanobridge defect per $268 \mu \mathrm{m}^{2}$ inspected area. Further efforts are needed to develop novel EUV chemically amplified resists to deliver high resolution and clean patterning even under drastic conditions of limited aerial image quality and photon-shot-noise.

\section{Acknowledgement}

The authors would like to thank Peter De Bisschop for the support on Stochalis software and John Biafore and Alessandro Vaglio Pret from KLA for the support on the ProLITH-Matlab script code.

\section{References}

1. A. Mallik A. Mallik, J. Ryckaert, A. Mercha, D. Verkest, K. Ronse, and A. Thean, Proc. SPIE, 9422 (2015) 94221N.

2. D. De Simone, Y. Vesters, A. Shehzad, G. Vandenberghe, P. Foubert, C. Beral, D. Van Den Heuvel, M. Mao, and F. Lazzarino, Proc. SPIE, 10143 (2017) 101430R.

3. D. De Simone, P. Vanelderen, and G. Vandenberghe, J. Photopolym. Sci. Technol., 30 (2017) 613.

4. P. De Bisschop, J. Micro/Nanolith. MEMS MOEMS, 16 (2017) 041013. 